\title{
Saúde da mulher: Medidas preventivas para o câncer de colo do útero
}

\author{
Women's health: Preventive measures for cervical cancer
}

Salud de la mujer: Medidas preventivas para el cáncer de cuello uterino

Rayssa Stéfani Sousa Alves ORCID: https://orcid.org/0000-0002-9666-675X Pontifícia Universidade Católica de Goiás, Brasil

E-mail: rayssastefani02@gmail.com

Francisco Lucas Leandro de Sousa

ORCID: https://orcid.org/0000-0003-2802-2378

Centro Universitário Maurício de Nassau, Brasil

E-mail: lucasleandro2912@gmail.com

Airton César Leite

ORCID: https://orcid.org/0000-0001-7184-8488

Centro Universitário Santo Agostinho, Brasil E-mail: ainton.cesar2014@gmail.com

Mariana Pereira Barbosa Silva

ORCID: https://orcid.org/0000-0003-0852-8099 Universidade Estadual do Piauí, Brasil E-mail: marianapbsilvaa@gmail.com

Ludymilla de Lima Silva

ORCID: https://orcid.org/0000-0001-7046-3543 Faculdade Evangélica de Ceres, Brasil E-mail: ludymillaludylls@gmail.com

Joelma Muniz Da Silva

ORCID: https://orcid.org/0000-0001-8242-7611 Faculdade Estácio de Teresina, Brasil E-mail: joelmamuniz19@hotmail.com

Laíssa Almeida Custódio da Silva ORCID: https://orcid.org/0000-0003-3115-9375 Centro Universitário Fametro, Brasil E-mail: laissa020@gmail.com

Israel Morais Martins

ORCID: https://orcid.org/0000-0001-6095-2319

Centro Universitário Maurício de Nassau, Brasil E-mail: israelmorais.m@hotmail.com

Rafael Mendonça Fonseca

ORCID: https://orcid.org/0000-0003-2862-5005

Universidade Federal do Maranhão, Brasil

E-mail: rafaelmendonca0902@gmail.com

Lucília da Costa Silva

ORCID: https://orcid.org/0000-0001-9386-5684

Centro Universitário Santo Agostinho, Brasil

E-mail: luciliafiso@outlook.com

Giselle Farias Medeiros

ORCID: https://orcid.org/0000-0002-6224-5316

Centro Universitário Maurício de Nassau, Brasil

E-mail: gi.ryan16@gmail.com

Brena Carolina Andrade Bordalo Sampaio ORCID: https://orcid.org/0000-0002-3551-8366 Escola Superior da Amazônia, Brasil E-mail: brenabsampaio@gmail.com

Janine Fragoso dos Santos ORCID: https://orcid.org/0000-0003-0627-7169 Instituto Pernambucano de Ensino Superior, Brasil E-mail: Janinefragoso06@gmail.com

Rebeca Dornelas Souza

ORCID: https://orcid.org/0000-0002-8977-3522 Universidade Federal de Goiás, Brasil

E-mail: rebecadornelas@discente.ufg.br

Lorena Vieira Fernandez de Araújo ORCID: https://orcid.org/0000-0002-9831-2306 


\begin{abstract}
Resumo
O estudo tem como objetivo compreender os fatores de risco para o câncer de colo do útero, e descrever as medidas preventivas para a doença. Trata-se de uma revisão integrativa de literatura, com abordagem qualitativa, realizada por meio da buscativa de artigos indexados na Biblioteca Virtual em Saúde (BVS), com o auxílio das seguintes bases de dados: Scientific Electronic Library Online (SCIELO), National Center for Biotechnology Information (PUBMED), Literatura Latino-Americana e do Caribe em Ciências da Saúde (LILACS). Os critérios de inclusão compreendem pesquisas de revisão da literatura disponíveis nos bancos de dados descritos, compreendidos entre os anos de 2010 a 2020. Como critérios de exclusão, não foram considerados artigos mediante a recompensação monetária, incompletos e não convergentes com este estudo. Para o levantamento dos dados foram analisados 10 artigos e 02 estudos referentes à entidades de saúde. Os resultados constatam que, os fatores de risco para o câncer de colo do útero compreende o início precoce das práticas sexuais, múltiplos parceiros íntimos, condições infecciosas e reativas, tabagismo ativo e passivo, uso prolongado de anticoncepcionais orais, e carências nutricionais. Concluímos a importância da percepção dos profissionais de saúde atuantes em unidades de Atenção Primária à Saúde, frente as orientações quanto as medidas preventivas para o câncer uterino, visando promover práticas assistenciais de acolhimento, e uma percepção qualificada para atender essa demanda, viabilizando o acolhimento inicial, acompanhamento integral, assistencial e humanizado.
\end{abstract}

Palavras-chave: Saúde da mulher; Colo do útero; Prevenção primária.

\title{
Abstract
}

The study aims to understand the risk factors for cervical cancer, and to describe preventive measures for the disease. It is an integrative literature review, with a qualitative approach, carried out by searching for articles indexed in the Virtual Health Library (VHL), with the help of the following databases: Scientific Electronic Library Online (SCIELO), National Center for Biotechnology Information (PUBMED), Latin American and Caribbean Literature in Health Sciences (LILACS). The inclusion criteria include literature review surveys available in the databases described, between the years 2010 to 2020 . As exclusion criteria, articles were not considered for monetary compensation, incomplete and not converging with this study. For data collection, 10 articles and 02 studies referring to health entities were analyzed. The results show that the risk factors for cervical cancer include the early onset of sexual practices, multiple intimate partners, infectious and reactive conditions, active and passive smoking, prolonged use of oral contraceptives, and nutritional deficiencies. We conclude the importance of the perception of health professionals working in Primary Health Care units, facing the guidelines regarding preventive measures for uterine cancer, aiming to promote welcoming care practices, and a qualified perception to meet this demand, enabling the initial reception, comprehensive, assistance and humanized monitoring.

Keywords: Women's health; Cervix; Primary prevention.

\section{Resumen}

El estudio tiene como objetivo comprender los factores de riesgo del cáncer de cuello uterino y describir las medidas preventivas para la enfermedad. Es una revisión integradora de la literatura, con enfoque cualitativo, realizada mediante la búsqueda de artículos indexados en la Biblioteca Virtual en Salud (BVS), con la ayuda de las siguientes bases de datos: Scientific Electronic Library Online (SCIELO), National Center de Información Biotecnológica (PUBMED), Literatura Latinoamericana y del Caribe en Ciencias de la Salud (LILACS). Los criterios de inclusión incluyen encuestas de revisión bibliográfica disponibles en las bases de datos descritas, entre los años 2010 a 2020. Como criterios de exclusión, los artículos no fueron considerados para compensación monetaria, incompletos y no convergentes con este estudio. Para la recolección de datos se analizaron 10 artículos y 02 estudios referidos a entidades de salud. Los resultados muestran que los factores de riesgo para el cáncer de cuello uterino incluyen el inicio temprano de prácticas sexuales, múltiples parejas íntimas, condiciones infecciosas y reactivas, tabaquismo activo y pasivo, uso prolongado de anticonceptivos orales y deficiencias nutricionales. Concluimos la importancia de la percepción de los profesionales de la salud que laboran en las unidades de Atención Primaria de Salud, frente a los lineamientos en materia de medidas preventivas del cáncer de útero, con el objetivo de promover prácticas de atención acogedora, y una percepción calificada para atender esta demanda, posibilitando la recepción inicial, seguimiento integral, asistencial y humanizado.

Palabras clave: La salud de la mujer; Cuello uterino; Prevención primaria.

\section{Introdução}

Segundo os estudos de Amaral, Gonçalves e Silveira (2017), o câncer do colo do útero (CCU) é considerado uma patologia de progressão lenta. Na fase benigna pré-invasiva as neoplasias inter-epiteliais da cérvice (NICs) são identificadas por lesões que se manifestam, já na fase maligna invasiva ocorre o desenvolvimento da lesão na cérvice, atingindo assim os tecidos externos do colo uterino e as glândulas linfáticas anteriores ao sacro. 
Ainda segundo os autores Amaral, Gonçalves e Silveira (2017), o câncer consiste em um conjunto de mais de cem doenças malignas com o crescimento irregular de células que acometem os órgãos e tecidos, podendo ter como origem as causas internas e/ou externas.

As células dividem - se rapidamente, de forma desordenada, agressiva e incontrolável, resultando no desenvolvimento de neoplasias malignas que podem se espalhar para outras partes do corpo (Amaral, Gonçalves \& Silveira, 2017).

O câncer do colo do útero é definido pela replicação desordenada do epitélio de revestimento, comprometendo o tecido subjacente (estroma), podendo invadir estruturas e órgãos contíguos ou à distância (INCA, 2017).

Há duas importantes classes de carcinomas invasores do colo do útero, de acordo com a origem do epitélio atingido: o carcinoma epidermóide sendo o tipo mais incidente e que acomete o epitélio escamoso, (compreendendo 90\% dos casos); e o adenocarcinoma, tipo mais raro e que acomete o epitélio glandular, (compreendendo 10\% dos casos) (INCA, 2017).

O câncer do colo do útero é uma doença crônica que pode desenvolver a partir de mudanças intraepiteliais em um tempo médio de cinco a seis anos, e se converter em um processo invasor, que se não for detectado precocemente pode provocar diversos danos ao organismo (INCA, 2017).

O câncer é uma doença de desenvolvimento lento, que pode se desenvolver silenciosamente, sem manifestar sintomas na fase inicial e evoluir para episódios de sangramento vaginal intermitente ou após a relação sexual, secreção vaginal irregular e dor abdominal associada com queixas urinárias ou intestinais em situações mais avançadas (INCA, 2017).

O câncer do colo do útero também conhecido como câncer cervical, é reputado como um grave problema de saúde pública no Brasil, em decorrência de sua evolução lenta e por exercer um importante impacto nas altas taxas de prevalência e na morbimortalidade de mulheres na fase reprodutiva de suas vidas (INCA, 2018).

Sendo assim, o câncer se desenvolve a partir de alterações no colo do útero, que está localizado no fundo da vagina. Essas lesões, nomeadas de lesões precursoras, são curáveis na maioria das vezes e podem levar anos para que se modifiquem e se tornem células cancerígenas, se não forem tratadas corretamente (Conceição et al., 2017).

No Brasil, o câncer do colo do útero é considerado a terceira causa de morte feminina, uma vez que, acometendo os tecidos adjacentes, alcança de forma impulsiva um determinado composto de células (Souza, Souto \& Santos, 2020).

Ainda assim, diversos fatores contribuem para a intensificação do Câncer Cérvico-Uterino, tendo como exemplo: relações sexuais precoce, relações sexuais com múltiplos parceiros, HPV, menarca, amenorreia, baixa condição socioeconômica, infecção genital, entre outros fatores (Souza, Souto \& Santos, 2020).

Carvalho, Domingos e Leite (2015) afirmam que, o câncer do colo do útero compreende cerca de $10 \%$ dos casos de cânceres na população feminina, correspondendo em torno de 500 mil novos casos por ano, o que por sua vez pode ser representado em duas mortes por minuto.

Atualmente, o câncer do colo do útero assume a quarta posição mundial entre as neoplasias malignas mais comuns entre as mulheres. Cerca de 527.000 casos foram identificados no ano de 2012, sendo a maioria nos países em subdesenvolvimento, onde essa doença ainda ocupa o segundo lugar das neoplasias malignas de maior prevalência, apresentando uma taxa de mortalidade de cerca de 268.000 óbitos por ano (Torre et al., 2015; Toye et al., 2017).

O elevado número de mortalidade está diretamente associado ao diagnóstico tardio da doença, relacionando-se aos mais diversos fatores, sendo, as dificuldades de acesso da população aos serviços de atenção primaria à saúde para adesão as medidas preventivas, e dificuldades dos gestores das unidades de saúde em estabelecer ações que envolvam vários níveis de atenção, integrando promoção, prevenção, diagnóstico e tratamento (Carvalho, Domingos \& Leite, 2015).

O público feminino representa um maior número na sociedade brasileira, elas são as que mais desfrutam dos serviços do Sistema Único de Saúde (SUS), tanto para próprio atendimento, quanto para seus filhos, familiares e pessoas mais próximas 
a elas. Dessa forma, a grande parte dos serviços oferecidos para as mulheres são voltados para a assistência materna, reprodução e enfermidades (Brasil, 2004).

O diagnóstico precoce do câncer cervical é de extrema importância para um tratamento adequado, com implementação de estratégias que visem o alcance, captação e minimização de danos à saúde da mulher, considerando a possibilidade de obter até $100 \%$ de cura (Rosa \& Labate, 2005).

Sendo assim, as orientações quanto as medidas preventivas para o câncer do colo do útero, realizado pela equipe de enfermagem na Atenção Primária de Saúde, são eficazes?

Este estudo tem como importância aprimorar o conhecimento acerca da assistência à saúde da mulher, sendo capaz também de despertar a ponderação acerca das medidas preventivas, educação em saúde, e as contribuições dos profissionais de saúde da Atenção Primária à Saúde. Sendo assim, o objetivo deste estudo consiste em compreender os fatores de risco para o câncer de colo do útero, e descrever as medidas preventivas para a doença.

\section{Metodologia}

Trata-se de um estudo de revisão integrativa da literatura, de caráter qualitativo. O levantamento de conteúdo foi realizado entre os meses de agosto a dezembro de 2020, através dos estudos publicados na Biblioteca Virtual em Saúde (BVS), com o auxílio das seguintes bases de dados: PubMed (National Center for Biotechnology Information), Lilacs (Literatura Latino-Americana e do Caribe em Ciências da Saúde), e Scientific Electronic Library Online (SciELO).

Foram utilizados os seguintes Descritores em Ciências da Saúde: Saúde da Mulher; Colo do Útero; Prevenção Primária; foi utilizado o operador booleano AND entre os descritores citados.

Os critérios de inclusão para a seleção dos conteúdos foram, artigos na linguagem portuguesa, inglesa e espanhola disponíveis nas bases de dados referidas, publicados na íntegra de acordo com a temática referente à revisão integrativa, documentos, regulamentações, e normativas de entidades de saúde compreendendo os anos de 2010 a 2020. Como critérios de exclusão, não foram considerados estudos mediante a recompensação monetária, relato de experiência, resumos, resenhas, artigos incompletos e não convergentes com este estudo.

\section{Resultados e Discussão}

Para compor os resultados deste estudo referentes as medidas preventivas para o câncer de colo do útero, foram encontradas 109 produções científicas com os descritores utilizados, sendo que apenas 47 estudos foram selecionados, 22 atenderam aos critérios de inclusão previamente estabelecidos. Desses, 12 foram excluídos com base nos critérios de exclusão. Restando 10 artigos para composição e analise do estudo. O fluxograma com o detalhamento das etapas de pesquisa está apresentado a seguir na Figura 1.

Além desses estudos, foram analisados 02 estudos referentes à entidades de saúde indexados nas bases de dados eletrônicas, acerca da temática proposta, sendo (Ministério da Saúde; Instituto Nacional de Câncer). 
Figura 1. Fluxograma de identificação e seleção dos artigos. 2020.

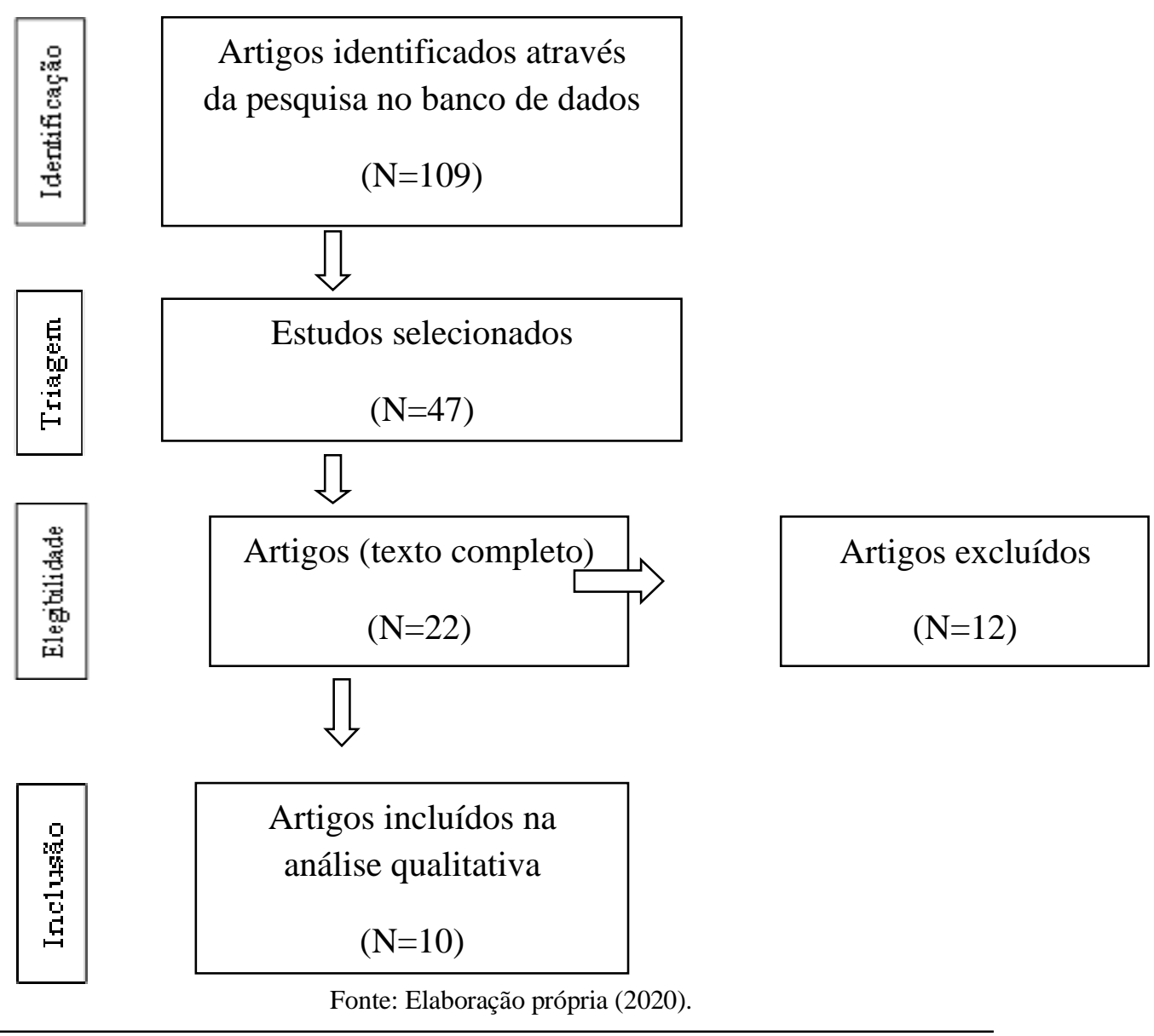

O câncer do colo do útero dificilmente desenvolve em mulheres que ainda não iniciaram a vida sexual, pois os principais fatores predisponentes para a doença compreende ao início precoce da vida sexual, múltiplos parceiros íntimos, e exposição a outras infecções sexualmente transmissíveis (Soares et al., 2010).

Soares et al. (2010) apresentam em seus estudos que, o câncer do colo do útero também pode estar associado à condições infecciosas e reativas, tabagismo ativo e passivo, uso prolongado de anticoncepcionais orais, e carências nutricionais.

O sedentarismo, tipo de alimentação, sobrecarga de responsabilidades, aumento considerável do número de mulheres chefes de família, competitividade, assédio moral e sexual no trabalho, são situações que podem estar relacionadas na ocorrência do câncer do colo do útero (INCA, 2017).

De acordo com os estudos de Zhang e Batur (2019), o rastreamento para o câncer do colo do útero deve ocorrer dos 21 aos 65 anos de idade, independentemente da situação vacinal e da iniciação sexual.

O rastreamento do câncer do colo do útero se baseia na história natural da doença e no discernimento do câncer se tratar de uma patologia muito invasiva, podendo progredir a partir de lesões precursoras (lesões intraepiteliais escamosas de alto grau e adenocarcinoma), que podem ser detectadas de forma precoce e tratadas corretamente, impedindo a evolução do câncer (Brasil, 2016).

A lesão no colo do útero é identificada através do exame preventivo, designado Papanicolau. Ainda nos dias de hoje, muitas mulheres não realizam o exame por medo, vergonha, ansiedade, ignorância, dificuldade de acesso aos Serviços de 
Saúde, falta de informação, e falta de confiança no profissional de saúde. Essas situações refletem na importância do profissional enfermeiro sobre sua atuação nesse contexto (Villani, 2016).

Segundo Oliveira et al. (2018), o Papanicolau é um exame simples, seguro e acessível, ofertado no Brasil gratuitamente nas Unidades Básicas de Saúde. O exame deve ser realizado em mulheres entre 25 a 64 anos de idade que já iniciaram as atividades sexuais, e deve ser realizado de $3 \mathrm{em} 3$ anos em casos de 2 resultados negativos.

O Papilomavírus Humano, mais conhecido como HPV, é um vírus da família Papavaviridae, potencialmente patogênico, de capacidade oncogênica, que é representada por induzir mutações genotípicas às células. O HPV pode ser classificado em baixo risco (DNA-BR) e alto risco (DNA-AR), as duas classificações podem provocar crescimento das células, porém, apenas os de alto risco podem evoluir para o carcinoma (Tanaka et al., 2019).

Wang et al. (2018) afirmam que, a vacina contra o HPV é fundamental para a proteção contra o câncer cervical, sendo o método mais eficaz para a prevenção do câncer uterino. No Brasil, a vacina disponível no Sistema Único de Saúde é a quadrivalente.

De acordo com Zhang e Batur (2019), no Departamento de Saúde e Recursos Humanos dos Estados Unidos existem três tipos de vacinas aprovadas, sendo: bivalente recomendada para os tipos 16 e $18 \mathrm{de} \mathrm{HPV}$, as quais possuem maior potencial carcinogênico. E a vacina quadrivalente direcionada para os tipos 16, 18, 6 e 11.

Uma outra opção disponível nos Estados Unidos é a Gardasil 9, que além de prevenir os tipos de HPV 16, 18, 06 e 11, também atua na prevenção dos tipos 31,33, 45, 52 e 58, considera - se, que esses nove tipos de HPV são responsáveis por 90\% dos cânceres de colo uterino, e grande parte das verrugas genitais são causadas por esse mesmo vírus (Lopes \& Ribeiro, 2019; Zhang \& Batur, 2019).

A vacina contra HPV proporciona 98\% de proteção contra o câncer do colo do útero, reduzindo drasticamente o risco de carcinoma cervical escamoso, adenocarcinomas e outras complicações causadas pela infecção do HPV. Contudo é de extrema importância que os profissionais de saúde forneça orientações para as famílias sobre a importância da vacinação (Zhang \& Batur, 2019).

As estatísticas indicam a incidência, a morbidade hospitalar e a mortalidade, consistindo em medidas de controle para a vigilância epidemiológica que possibilitam analisar a ocorrência, a distribuição e o desenvolvimento das doenças. A base para a construção desses indicadores são os números provenientes, principalmente, dos Registros de Câncer e do Sistema de Informações sobre Mortalidade (INCA, 2019).

O profissional enfermeiro tem desempenho direto na promoção e prevenção do câncer colo uterino, desenvolvidas a partir da consulta de enfermagem, realização do exame colpocitológico, controle da qualidade do exame, investigação, interpretação e avaliação dos resultados e o encaminhamento necessário no momento propício de uma consulta (Brandão, Andrade, \& Olivindo 2020).

No cotidiano das equipes da Estratégia Saúde da Família, os enfermeiros estão engajados em todas as atividades de prevenção do câncer do colo do útero. As coletas citológicas são executadas prioritariamente por enfermeiros em nível de Atenção Primária à Saúde. Assim, a enfermagem vem se destinguindo dia após dia na tarefa do cuidado preventivo do câncer de colo do útero, visando desenvolver estratégias que incentivem e mobilizem os profissionais envolvidos para a realização integral desse cuidado (Mendes, Mesquita \& Lira, 2015).

As ações para a prevenção do câncer de colo do útero ocorrem por meio de ações de educação em saúde, vacinação de grupos indicados e deteç̧ão precoce do câncer. Além da identificação de suas lesões precursoras mediante seu rastreamento (Brasil, 2016).

A educação em saúde para o câncer de colo do útero compreende a realização de campanhas educativas voltadas para a prevenção de doenças e agravos físicos e mentais do indivíduo. Sendo primordial o conhecimento, e a busca de auxílios de 
unidades de saúde e centros educativos, que valorizam estratégias construtivas principalmente na cobertura de comunidades, apontando as principais necessidades de ações voltadas para públicos alvo, influenciando na detecção precoce da patologia e diminuindo os casos de óbitos no Brasil (Melo et al., 2012).

É de suma importância que os enfermeiros tenham uma visão holística, integral e humanizada, pois o controle do câncer de colo do útero relaciona - se, a ações voltadas para a saúde, prevenção do câncer e qualidade de vida, assim o enfermeiro intervém nessas ações, e em visitas domiciliares e consultas de enfermagem de forma integralizada (Amaral, Gonçalves \& Silveira, 2017).

O desenvolvimento de ações efetivas no controle do câncer de colo do útero, é muito importante o envolvimento do enfermeiro com os outros profissionais da Estratégia Saúde da Família, a fim de utilizarem os conhecimentos epidemiológicos, fatores de risco, sinais, sintomas e instrumentos existentes para a prevenção do referido câncer (Ramos et al., 2014).

É necessário ainda, que haja uma real preocupação com a gravidade da doença por parte de todos os responsáveis nos níveis Federais, Estaduais e Municipais, visando garantir o acesso aos Serviços de Saúde, e uma assistência de qualidade proporcionada por profissionais qualificados, em uma infraestrutura adequada (Ramos et al., 2014).

É imprescindível que os profissionais de saúde, principalmente os enfermeiros, tenham seu olhar voltado para o controle da doença, pois a morbimortalidade pode ser reflexo de ações e políticas de prevenção deficitárias. Além disso, vencer as barreiras para uma melhor adesão da mulher ao exame preventivo significa dar atenção aos relatos e experiências de quem faz o exame para identificar os significados para as mulheres que a ele se submetem, de modo à retirar informações e argumentos para planejar e adequar às orientações de prevenção (Sementille \& Queiroz, 2013).

Como medida preventiva para o $\mathrm{CCU}$, as mulheres devem ser orientadas sobre os comportamentos de risco, sinais de alerta e a frequência da busca pelos serviços. A consulta de enfermagem tem papel fundamental na aproximação da paciente, pois durante sua realização a mulher adquire confiança e segurança, o que facilita a troca informações importantes para detecção de possíveis problemas que afetam a saúde e a qualidade de vida (Sementille \& Queiroz, 2013).

Os enfermeiros utilizam métodos científicos para a identificação de situações relacionadas ao processo saúde/doença, além de prescrever e implementar medidas que contribuam para a promoção, prevenção, proteção da saúde, recuperação e reabilitação do indivíduo, família e comunidade (Sementille \& Queiroz, 2013).

\section{Considerações Finais}

Os resultados encontrados neste estudo demonstraram que o câncer de colo de útero é um dos temas mais abordados atualmente, devido ao fato de ser uma das neoplasias que mais acometem as mulheres.

O câncer de colo uterino tem cura, desde que seja descoberto em sua fase inicial, assim, a assistência integral a saúde da mulher deve abranger um conjunto de ações que envolvem a promoção, a prevenção, o diagnóstico, o tratamento e a reabilitação. Dentre estas ações, estão àquelas voltadas para o controle dos cânceres do colo do uterino, principalmente as campanhas preventivas ministradas em campos de saúde.

Além do exame Papanicolau, a prevenção deste tipo de patologia deve se basear em medidas educativas por intermédio da educação sexual e de orientações sobre como se desenvolve a doença, quanto ao uso correto de preservativos e da importância do autocuidado e da valorização do próprio corpo.

Este estudo evidenciou a necessidade de melhoria na informação sobre o assunto, projetos de intervenção podem ser pensados para levar ao conhecimento das condutas preventivas para o público, incluindo uma revisão nos programas de disseminação de informações e prevenções existentes que possam resultar em mudanças de atitudes da população. 
Evidenciou-se no estudo, que a enfermagem desenvolve ações em saúde que incidam sobre tal problemática, tais como: criação de espaços para informações / reflexão sobre o corpo, sexualidade, autocuidado e o exame citopatólogico dirigido às mulheres.

O profissional enfermeiro atua, também, no momento da realização do exame preventivo, orientando e sensibilizando as mulheres desde o momento da marcação e na realização do exame Papanicolau, esclarecendo questões relevantes como as condições necessárias para o momento de realização do exame e o tempo certo para repeti-lo.

\section{Referências}

Amaral, M. S., Gonçalves, A. G., \& Silveira, L. C. G. (2017). Prevenção do câncer de colo de útero: a atuação do profissional enfermeiro nas unidades básicas de saúde. Revista Científica FacMais, 8(1).

Brandão, A. M. R., de Andrade, F. W. R., \& de Olivindo, D. D. F. (2020). Atuação do enfermeiro da estratégia da saúde da família no manejo da mulher com resultado de colpocitologia alterado. Research, Society and Development, 9(10), e5899108962-e5899108962.

Brasil. (2004). Política nacional de atenção integral à saúde da mulher: princípios e diretrizes / Ministério da Saúde, Secretaria de Atenção à Saúde, Departamento de Ações Programáticas Estratégicas. - Brasília: Ministério da Saúde.

Brasil. (2016). Política nacional de atenção integral à saúde da mulher: princípios e diretrizes / Ministério da Saúde, Secretaria de Atenção à Saúde, Departamento de Ações Programáticas Estratégicas. - Brasília: Ministério da Saúde.

Carvalho, B. G., Domingos, C. M., \& Leite, F. S. (2015). Integralidade do cuidado no Programa de Controle do Câncer de Colo Uterino: visão das usuárias com alteração na citologia oncótica. Saúde Debate, 39(106):707-717.

Conceição, J. P. S., Medeiros, M. M. S., Rodrigues, L. M. S., Bráz, M. R., Balbino, C. M., \& Silvino, Z. R. (2017). O conhecimento do enfermeiro sobre a prevenção do câncer de colo de útero na atenção básica. Revista Enfermagem Atual In Derme, 2017.

Ercole, F. F., Melo, L. D., \& Alcoforado, C. L. G. C. (2014). Revisão integrativa versus revisão sistemática. Rev Min Enferm, 18(1), 9-12.

Instituto Nacional de Câncer (2017). Controle do câncer do colo do útero: Fatores de risco. INCA; Ministério da Saúde, 2017.

Lopes, V. A. S., \& Ribeiro, J. M. (2019). Fatores limitadores e facilitadores para o controle do câncer de colo de útero: uma revisão de literatura. Revista Ciência \& Saúde Coletiva, v.24, n.9.

Melo, M. C. S. C., Vilela, F., de Oliveira Salimena, A. M., \& de Oliveira Souza, I. E. (2012). O enfermeiro na prevenção do câncer do colo do útero: o cotidiano da atenção primária. Revista Brasileira de Cancerologia, 58(3), 389-398.

Mendes, Y. L. C., de Mesquita, K. O., \& Lira, R. C. M. (2015). Prevenção do câncer de colo uterino: analisando a atuação do enfermeiro da atenção primária à saúde. SANARE-Revista de Políticas Públicas, 14(2).

Oliveira, M. M., Andrade, S. S. C. A., Oliveira, P. P. V., Silva, G. A. Silva, M. M. A., Malta, D. C. (2018). Cobertura de exame Papanicolaou em mulheres de 25 a 64 anos, segundo a Pesquisa Nacional de Saúde e o Sistema de Vigilância de Fatores de Risco e Proteção para Doenças Crônicas por Inquérito Telefônico, 2013. Revista Brasileira de Epidemiologia. 21.

Ramos, A. L., da Silva, D. P., Machado, G. M. O., Oliveira, E. N., \& dos Santos Lima, D. (2014). A atuação do enferme iro da estratégia saúde da família na prevenção do câncer de colo de útero. SANARE-Revista de Políticas Públicas, 13(1).

Rosa, W. A. G., Labate, R. C. (2005). Programa Saúde da Família: A Construção de um Novo Modelo de Assistência. Rev. Latinoam Enfer. São Paulo, 2005.

Sementille, E. C., \& Queiroz, F. C. (2013). Atuação do enfermeiro na saúde da mulher: prevenção do câncer do colo do útero. Ensaios e Ciência, 17(1).

Soares, M. C., Meincke, S. M. K., Mishima, S. M., Simino, G. P. R. (2010). Câncer de colo uterino: caracterização das mulheres em um município do sul do Brasil. Esc Anna Nery Rev Enf. jan/mar; 14 (1): 90-96.

Souza, S. A. N., Souto, G. R. \& Santos, M. W. L., (2020). Assistência da enfermagem relacionada ao câncer uterino. Revista JRG De Estudos Acadêmicos, 3(6), 04-11.

Tanaka, E. Z., Kamizaki, S. S., Quintana, S. M., Pacagnella, R. C., Surita, F. G. (2019). Knowledge of pregnant adolescents about humanpapillomavirus. Rev Bras Ginecol Obstet. 41(5): 291-297.

Torre, L. A., Bray, F., Siegel, R. L., Ferlay, J., Lortet-Tieulent, J., Jemal A. (2015). Global cancer statistics, 2012. CA Cancer J Clin. 65(2):87-108.

Toye, M. A., Okunade, K. S., Roberts, A. A., Salako, O., Oridota, E. S., Onajole, A. T. (2017). Knowledge, perceptions and practice of cervical cancer prevention among female public secondary school teachers in Mushin local government area of Lagos State, Nigeria. Pan Afr Med J. $10(28), 221$.

Zhang, S., Batur, P. (2019). Human papillomavirus in 2019: An update on cervical cancer prevention and screening guidelines. Cleveland Clinic Journal of Medicine, 86(3). 